\title{
EL AUTORRECONOCIMIENTO ÉTNICO COMO DETERMINANTE EN LA SATISFACCIÓN LABORAL: UN ESTUDIO EMPÍRICO PARA SANTIAGO DE CALI, 2013
}

\section{THE ETHNIC SELF-RECOGNITION AS A DETERMINANT OF JOB SATISFACTION: AN EMPIRICAL STUDY FOR SANTIAGO DE CALI, 2013}

\author{
Geovanny Castro-Aristizabal ${ }^{1}$ \\ Diana Constanza Camacho Jiménez ${ }^{2}$ \\ Jeinson Fabián Prado Flórez ${ }^{3}$
}

Recibido: 02/10/2020

Aprobado: 09/12/2020

\begin{abstract}
RESUMEN
En este trabajo se analiza si el autorreconocimiento étnico es un determinante sobre la satisfacción laboral de la población trabajadora de la ciudad de Santiago de Cali, también se evalúa la incidencia de otras variables demográficas y las características del empleo. Se estiman un modelo logit ordenado y un probit ordenado a partir de la Encuesta de Empleo y Calidad de Vida -EECV- para el Municipio de Santiago de Cali, realizada por el Ministerio de Trabajo y la Alcaldía de Santiago de Cali, en el período de noviembre 2012 a enero 2013. Los resultados muestran que considerarse afrodescendiente no incide significativamente sobre sentirse o no satisfecho con el empleo; sin embargo, cuando se evalúa la variable raza de manera conjunta (incluyendo todos los grupos étnicos) esta variable influye en la satisfacción laboral. Igualmente, se evidencia que factores como la edad, el estrato, el estado civil, el medio de transporte para ir al trabajo y la calidad del empleo son determinantes en el nivel de satisfacción que un caleño siente con su empleo.
\end{abstract}

PALABRAS CLAVE: SATISFACCIÓN LABORAL, AUTORRECONOCIMIENTO ÉTNICO, MODELO LOGIT ORDENADO, MODELO PROBIT ORDENADO, CALI, COLOMBIA

CLASIFICACIÓN JEL: C25, D01, J15, J28

\footnotetext{
1 Universidad Autónoma de Bucaramanga; Código Postal: 680003; Bucaramanga, Colombia; gcastro793@unab.edu.co

2 Inspira Research SAS; Código Postal: 760044; Valle del Cauca, Colombia; diana.ccj@hotmail.com

3 Alcaldía de Palmira; Código Postal: 763533; Valle del Cauca, Colombia; fabianprado.flz@gmail.com
} 


\section{ABSTRACT}

This paper analyzes if the ethnic self-recognition is a determinant of satisfaction job for the workers of the city of Santiago de Cali; the incidence of other demographic variables and employment characteristics are also evaluated. An ordered logit model and an ordered probit model are estimated from the Employment and Quality of Life Survey (Encuesta de Empleo y Calidad de Vida -EECV)- for the Municipality of Santiago de Cali, conducted by the Ministry of Labor and the Mayor's Office of Santiago de Cali, in the period November 2012 to January 2013. The results show that considering afro-descendent people does not significantly affect whether or not they feel satisfied with the job, however when it is evaluated together with other ethnic groups, this variable as a whole seems to influence job satisfaction. Likewise, it is evident that factors such as age, stratum, marital status, means of transportation to go to work and the quality of employment are determining factors in the level of satisfaction that Caleños feels with their jobs.

KEYWORDS: JOB SATISFACTION, RACE, ORDERED LOGIT MODEL, ORDERED PROBIT MODEL, CALI, COLOMBIA.

JEL CLASSIFICATION: C25, D01, J15, J28.

\section{INTRODUCCIÓN}

La satisfacción laboral se puede definir como la manifestación de las emociones, sentimientos o respuestas afectivas, ya sean favorables o desfavorables, de la persona trabajadora hacia su empleo. Desde la rama de la psicología de las organizaciones, Chiang Vega y Ojeda Hidalgo (2013) indican que este concepto ha sido abordado desde diferentes enfoques, que se pueden clasificar en dos grupos principales: el primero, hace referencia al estado "emocional, sentimientos y respuestas afectivas", dentro de este grupo está la definición dada por Locke (1976) que la define como un estado emocional resultante de la percepción subjetiva de las experiencias laborales de las personas.

El segundo grupo concibe la satisfacción laboral como "una actitud generalizada ante el trabajo", como la expuesta por Griffin y Bateman (1986), quienes la definen a través de características específicas como el trabajo, el sueldo, la supervisión, los beneficios, las oportunidades de promoción, las condiciones de trabajo, los compañeros y las prácticas de la empresa. En este último grupo, también se encuentra la interpretación hecha por Bravo et al. (1996), definiendo la satisfacción como las actitudes que la persona desarrolla ante su empleo, ya sea de manera general o ante facetas específicas. En este sentido, tal y como lo exponen los autores, la satisfacción laboral es un elemento clave para el trabajador, ya que a medida que una persona se siente más satisfecha en su lugar de trabajo, aumenta la productividad y se reducen las necesidades de buscar otras opciones laborales.

Basado en las definiciones anteriores, es posible decir que el concepto de satisfacción laboral involucra valoraciones propias, es decir, cuenta con un alto componente subjetivo, ya que dos personas en un mismo empleo $y$ en una misma empresa pueden tener una percepción diferente frente a la satisfacción que perciben sobre su trabajo. Estas valoraciones subjetivas han sido motivo de estudio de ciencias como la psicología y la sociología.

Por otra parte, en el área de la Economía, quienes son más afines a enfoques tradicionales se oponen al estudio de la satisfacción laboral, pues la teoría económica basa sus mediciones de la utilidad o satisfacción del individuo, en las elecciones observables hechas por ellos mismos, y los juicios personales incluidos dentro de este tipo de estudios (satisfacción laboral) no son objetivamente observables. A modo de ejemplo, Stiglitz (2002) plantea que fundamentar las 
elecciones de los individuos respecto a su empleo, solo teniendo en cuenta las utilidades derivadas del consumo a través del salario recibido, y el costo que esto trae medido en horas de ocio, está muy alejado de la realidad laboral actual.

En este orden de ideas, un aspecto determinante en la satisfacción laboral corresponde a la calidad del empleo. Farné (2003) afirma que este último concepto se fundamenta en consideraciones objetivas, a diferencia de la satisfacción laboral que es una medida subjetiva. Sin embargo, Reinecke y Valenzuela (2000) definen la satisfacción laboral como la percepción de las características objetivas de la calidad del empleo, las cuales pondera la persona trabajadora según sus preferencias y expectativas, es decir, incorporan un análisis de equivalencias subjetivas de la percepción de calidad del empleo de cada individuo. Así pues, el estudio de este factor tiene gran relevancia en la economía laboral, dado que tiene un efecto trascendental en el bienestar de la población trabajadora. Pese a lo anterior, en la actualidad, para evaluar la calidad de un empleo, se tiene en cuenta las características intrínsecas de este, las cuales deben ser objetivas.

Como se puede inferir de las consideraciones anteriores, la persona trabajadora tiene en cuenta no solo los beneficios pecuniarios, sino también los no pecuniarios propios de su empleo, para evaluar su satisfacción laboral. Desde este contexto, las variables que impactan en esta pueden dividirse en varios grupos: las características demográficas o individuales (edad, género, nivel educativo, raza), las extrínsecas al trabajo o características del empleo (salario, tipo de contrato, horas de trabajo, posición ocupacional, lugar de trabajo, oportunidad de promoción, satisfacción con la tarea, entorno laboral) y las intrínsecas al trabajo o indicadoras de la calidad del ajuste laboral (antigüedad, desajuste educativo, calidad de la enseñanza, especialidad de la enseñanza, tiempo en desempleo).

Basado en el abordaje teórico y empírico del estudio de satisfacción laboral, en el ámbito internacional se han desarrollado investigaciones que no representan lineamientos definidos claramente para el análisis de este fenómeno. Autores como Pujol-Cols y Dabos (2018) para el caso de Argentina, se enfocaron principalmente en identificar los factores determinantes, a partir de la revisión de estudios de caso. Por su parte, García (2003) realizó un análisis econométrico que buscaba identificar la educación como principal determinante de la satisfacción laboral en España. Somarriba Arrechavala, et al. (2007) realizan un análisis descriptivo para la comunidad de Castilla y León, en España, asociando la calidad del empleo como proxy de la satisfacción laboral. Borra Marcos y Gómez García (2012) hacen un estudio econométrico en Sevilla, España, para identificar la satisfacción de la población trabajadora, teniendo en cuenta el impacto de ciertas características sobre el salario. En líneas similares de investigación, se ubica lo realizado por Chiang Vega y Ojeda Hidalgo (2013) y Gamero Burón (2005).

Por otra parte, en el caso particular de Colombia, el Observatorio Laboral LaboUR (2018) efectúa un análisis de tipo descriptivo, el cual caracteriza la satisfacción laboral en las diferentes áreas metropolitanas sin intentar explicarlas. Otros tipos de estudios han empleado la estimación de diferentes modelos econométricos, utilizando variables del entorno laboral, los ingresos, las características socieconómicas e inclusive relacionando la calidad del empleo como factor asociado a la satisfacción laboral (Amezquita Ospina, 2013; Angulo Pico et al., 2014; Barrera Barrios \& Botia Díaz, 2014; Botello Peñaloza \& Ríos, 2014; Olarte, 2011; Ruíz Tavera, 2013). A nivel local, en la ciudad de Santiago de Cali, los estudios se han enfocado en la determinación de los factores que inciden en la calidad del empleo, más que en la satisfacción laboral. Tal es el caso de los trabajos realizados por Arroyo Mina et al. (2016), y Bustamante y Arroyo Mina (2008), quienes indagan sobre de la incidencia de características como la raza, sobre la calidad del empleo.

Ahora bien, todos los trabajos anteriormente enunciados, tienen en común que, para explicar la satisfacción laboral, utilizan variables demográficas de tipo extrínseca o intrínseca, entre ellas se incluyen la calidad del trabajo como factor determinante. No todos los estudios se desarrollan bajo un único grupo, sino al contrario, presentan combinaciones ligadas a la calidad y cantidad de 
datos disponibles para realizar el análisis; por lo tanto, resultan escasos los estudios que relacionen o cuantifiquen el impacto que tiene la etnia o raza, sobre la satisfacción laboral, de hecho, dentro de la revisión de la literatura, solo se halló el trabajo realizado por Diano (2016), quien encontró una relación negativa entre la condición de afrodecendencia en Uruguay y la satisfacción laboral, ello gracias a la discriminmación racial, lo que lleva a que el ambiente laboral no sea el adecuado, y consecuentemente, la población afrodecendiente manifieste su descontento en sus lugares de trabajo. Ya para el caso particular de Colombia, solo se encontró el trabajo realizado por Rivas Oyuela (2014), quien analiza la población informal de la ciudad de Cali, asociando la satisfacción laboral, con la experiencia, el sector económico y la etnia, además de una serie de variables de tipo socioeconómico.

Dado lo anterior, el presente trabajo tiene como objetivo central identificar cuáles son los principales aspectos que influyen en la satisfacción laboral en Santiago de Cali, para el año 2012. Específicamente, se desea determinar si el autorreconocimiento étnico es un factor condicionante de dicha satisfacción. Incluir la condición de autorreconocimiento étnico se constituye como un elemento diferenciador de los demás estudios realizados en este ámbito, más en el caso del estudio en el contexto local. Para el logro de los objetivos planteados aquí, se utilizarán los datos de la Encuesta de Empleo y Calidad de Vida -EECV- para el Municipio de Santiago de Cali, realizada por el Ministerio de Trabajo y la Alcaldía de Santiago de Cali, en el periodo noviembre 2012 a enero 2013. Se emplearán variables socioeconómicas y de percepción del individuo, e igualmente se utilizará como variable explicativa el índice de la calidad del empleo, construido con base en el índice sintético propuesto por Farné (2003), fundamentado en cuatro variables básicas: el ingreso, la modalidad de contratación, la afiliación a la seguridad social y el horario de trabajo.

Estudiar el autorreconocimiento étnico y su efecto sobre la satisfacción laboral nace de la premisa de que Cali, por su ubicación y por su contexto histórico, cuenta con una proporción importante de población que se autorreconoce como negra, ya que, de acuerdo con el censo realizado por el Departamento Administrativo Nacional de Estadística -DANE- en el año 2005, en la ciudad de Cali, 542.039 personas se identifican como negro, mulato o afrocolombiano, lo que representa el $26,12 \%$, razón por la cual, se prestará especial atención a esta variable, buscando determinar la existencia de alguna relación entre etnia y satisfacción laboral.

El presente trabajo se ha estructurado en cinco secciones. Seguido de esta introducción, en la sección dos, se muestra el marco de referencia, en el que se expone una revisión de la literatura sobre las principales teorías del mercado laboral relacionadas con la satisfacción laboral y las diferentes investigaciones que se han llevado a cabo sobre el tema a nivel internacional, nacional y local. La tercera sección describe los instrumentos metodológicos necesarios para el logro de los objetivos. En la cuarta sección, se muestran los resultados del modelo empleado y finalmente se exponen las conclusiones y recomendaciones del estudio.

\section{MARCO DE REFERENCIA}

\section{Marco teórico}

Cuando una persona ofrece sus servicios laborales a un empresario, no solo le importa la remuneración monetaria (directa), sino que también tiene en cuenta las características no monetarias del cargo al cual aspira, tal y como la seguridad, la dureza del trabajo, la estabilidad del empleo y las oportunidades de formación y de ascenso (McConnell \& Brue, 1997), ello se debe a que el trabajo remunerado, además de proporcionar los medios materiales necesarios para sobrevivir y disfrutar la vida (Gamero Burón, 2005), también "representa la actividad individual más intensa, 
temporalmente más amplia y física, cognitiva y emocionalmente más exigente e influyente de la vida" (Wein, 1985, citado en Gamero Burón, 2005, p. 8), ocupando gran parte del tiempo de las personas, por lo que su bienestar va a estar condicionado a factores relacionados tanto con su vida personal como con el aspecto laboral.

Como se sabe, "la economía es la ciencia que estudia la conducta humana como una relación entre fines y medios escasos que tienen usos alternativos" (Robbins, 1932, p. 15) y de acuerdo con este enfoque, los individuos activos en el mercado laboral toman decisiones respecto a sus empleos (seguir en el empleo, aceptar uno nuevo, abandonarlo), afectando los recursos de los que disponen, esto es, horas de ocio, con el objetivo de maximizar sus posibilidades de consumo (de bienes, servicios y ocio). Una de las decisiones que deben tomar las personas trabajadoras es cómo deben distribuir su tiempo, dada la escasez de éste, entre trabajo (el tiempo dedicado a un empleo remunerado) y el ocio (todas aquellas actividades por las que la persona no es remunerada: trabajo doméstico, tiempo dedicado al consumo, educación, desplazamientos, descanso). Para elegir la actividad a la que dedicarán más tiempo, realizan un análisis de los costos y los beneficios, comparando la utilidad adicional (medida a través de la renta) generada por una hora adicional de trabajo, con el valor del ocio perdida. Este modelo se conoce como consumo-ocio (también llamado renta-ocio). Entre más tiempo dedique una persona a una de estas actividades, menos tendrá para la otra, $y$ a esta pérdida se le llama costo de oportunidad.

Ahora, dentro del análisis tradicional neoclásico del mercado laboral, el trabajo es visto como un "mal", ya que, si bien, a mayor cantidad de horas trabajadas, mayor será el nivel de ingreso del individuo y, por lo tanto, podrá adquirir más bienes y servicios, igualmente, mayor será su desutilidad por laborar, pues al trabajar más tiempo, deberá sacrificar más horas de ocio. Por el contrario, a menos horas de trabajo, el individuo tendrá mayor utilidad del ocio, pero menor renta, lo que se traduce en un menor consumo. El individuo entonces elegirá entre distintas combinaciones de renta (consumo) y ocio, las cuales le reportarán el mismo nivel de utilidad.

Sin embargo, si bien la concepción neoclásica caracteriza el trabajo como un coste (mal), cuya única motivación es la renta, existen otras líneas de investigación que se alejan de esta idea, aceptando la existencia de individuos que perciban un bienestar en su trabajo, a partir del aporte de este a sus vidas, lo cual se asemeja a lo dicho por Lancaster (1971), quien manifiesta que a los consumidores no les importa la posesión de un bien, si no sus posibles beneficios. A partir de ello, se plantea que el individuo puede obtener una utilidad directa del trabajo, basado en otros aspectos adicionales al salario, con lo que alcanzaría la categoría de "bien normal", cuyo "consumo" se asocia con el logro de la dignidad humana. El trabajo no solo ofrece una renta, sino también entretenimiento, contactos sociales, prestigio, entre otros, lo cual se traduce también en utilidad para el individuo (Scitovsky, 1976).

Tomando como base esta concepción teórica, se generan dos temas de importancia para la economía laboral: el estudio de la calidad del empleo y de la satisfacción laboral, siendo el último de estos, el que será tratado en la presente investigación. La satisfacción laboral no es equivalente a la calidad del empleo. Esta última se relaciona con factores objetivos, que reflejan la realidad tal como es y no dependen de las percepciones de los trabajadores (Bustamante \& Arroyo Mina, 2008), mientras que la satisfacción laboral se refiere a factores subjetivos, por lo que tendrá en cuenta las preferencias individuales del trabajador y cómo ciertos aspectos impactan sobre su utilidad.

La satisfacción laboral, por lo tanto, toma como base la evaluación directa que el individuo hace de su empleo, lo cual implica juicios personales con los que la teoría económica estándar no está de acuerdo, puesto que no son posibles de medir a través de variables observables, Incluso, Rose (2000) considera que se debe suponer racionalidad en la evaluación que un trabajador realiza sobre la satisfacción en su empleo, un supuesto muy conveniente para la economía neoclásica, pues de esta manera se evitaría la incorporación de aspectos emocionales en este tipo de estudios, eludiendo la generación de ruido que no permitiría realizar un buen análisis, según la economía tradicional. 
Adicionalmente, la satisfacción laboral es definida como "la actitud general del individuo hacia su trabajo" (Robbins, 2004, p. 72). La actitud supone que la persona trabajadora evaluará su experiencia en su empleo como bueno o malo, agradable o desagradable. "Se trata de una evaluación que afecta a la esfera del pensamiento, pero también a los sentimientos y a la determinación comportamental de la persona" (Chiang Vega \& Ojeda Hidalgo, 2013, p. 43), lo cual va en concordancia con lo expresado por Pastor Ramos (2000), quien considera que, al evaluar la satisfacción laboral, el individuo manifiesta su actitud hacia el trabajo, y su valoración no solo estará influida por su parte afectiva (emociones), sino también por la esfera cognitiva (pensamiento) $y$ las disposiciones conductuales.

Otras definiciones, en cambio, ubican el foco de su análisis en alguno de los dos aspectos, este es el caso de Katzel (1964) que da mayor importancia al aspecto cognitivo, al precisar que la satisfacción laboral es "la expresión verbal de la evaluación por parte de un individuo de su empleo" y el de Price y Mueller (1986) quienes la describen como "el grado en el que a los individuos les gusta su trabajo", otorgándole mayor peso al componente afectivo. En general, los individuos evalúan de manera continua la situación actual de sus necesidades y la capacidad que tienen sus empleos de satisfacerlas. Cuando se presentan divergencias entre estas dos, los individuos se sienten insatisfechos con su trabajo, de lo contrario, estarán satisfechos laboralmente.

Como se evidencia en lo dicho anteriormente, los modelos teóricos se basan en características subjetivas del individuo para explicar la satisfacción laboral; pese a ello, desde la Economía, se han realizado ejercicios empíricos, algunos de los cuales se expondrán posteriormente, que intentan analizar el impacto de variables independientes sobre la satisfacción de la población trabajadora con su empleo.

\subsection{Revisión de literatura}

Los estudios que se han desarrollado para explicar la satisfacción laboral se han abordado desde diferentes modelos teóricos, ciencias y enfoques metodológicos. A continuación, se realizará la presentación de algunas investigaciones con el propósito de conocer como se ha abordado esta problemática desde los diferentes autores.

En el ámbito internacional, existen una gran variedad de estudios; recientemente se destaca el desarrollado por Pujol y Dabos (2018) quienes realizaron una revisión de la literatura empirica para el periodo 1996-2016, con el fin de identificar los principales factores determinantes de la satisfacción laboral. La revisión de los autores clasificó los determinantes en tres categorias de estudio: 1) los determinantes situacionales; que se basa en características propias del trabajo (habilidades, tarea, identidad, autonomia y retroalimentación), 2) los determinantes disposicionales, que se definen como características inobservables propias del individuo (extraversión, responsabilidad y neuroticismo) y 3) los efectos interaccionistas que articulan los determinantes situacionales y disposicionales.

Por otra parte, el estudio desarrollado por Diano (2016) indaga si la motivación y satisfacción laboral de los trabajadores afrodescendientes se ven afectadas ante la percepción de racismo en el ámbito laboral. Para desarrollar este objetivo, el autor aplicó un cuestionario semidirigido a integrantes de organizaciones de la comunidad afrouruguaya y los resultados están enfocados hacia la produccion de conocimiento académico en la campo de la psicología laboral y motivacional. Borra y Gómez (2012) buscan responder si la renta laboral compensa las condiciones no monetarias del trabajo, obteniendo como resultado que el salario es exógeno en la ecuación de satisfacción laboral y tiene un efecto directo sobre la misma. Por otra parte, los autores encuentran que existen condiciones no monetarias que impactan negativamente al trabajador como son gran cantidad de horas semanales sin contraprestación y laborar en un empleo de alto riesgo, situaciones que no son compensadas salarialmente. Así mismo, Lydon y Chevalier (2002) analizan el impacto del 
salario en la satisfacción laboral. Los resultados permitieron concluir que cuando los salarios son tratados como endógenos, el impacto del salario sobre la satisfacción laboral se duplica respecto a lo obtenido cuando es tratado como exógenos.

Por su parte, Cassar (2010) investigó los determinantes de la satisfacción laboral en Chile, haciendo uso de un modelo Logit Ordenado, y encontró que el determinante más importante de satisfacción laboral para los chilenos es el ingreso, lo cual es congruente con lo que ocurre en los países industrializados. Asimismo, halla que las mujeres están más satisfechas con su empleo que los hombres y que el número de horas trabajadas no parece tener un impacto sobre la satisfacción laboral. Además de lo anterior, el autor encontró que en igualdad de condiciones, para todos los trabajadores chilenos la variable de riesgo laboral tiene un impacto negativo sobre la satisfacción laboral y la protección laboral un efecto positivo, sin embargo, al controlar el efecto de estas dos variables, los trabajadores independientes chilenos están más satisfechos con su trabajo que los empleados chilenos, lo que muestra la deficiente protección y seguridad laboral que tienen los trabajadores independientes en países en desarrollo como Chile.

Gamero (2007) realiza una investigación para los trabajadores formales en España e incorpora variables como: relaciones personales en el centro de trabajo, perspectivas de futuro, conciliación entre lo laboral y familiar; actividad económica de la empresa. Los resultados permiten concluir que entre más bajas sean las expectativas del individuo respecto a su trabajo, es más probable que se declare satisfecho, además, el género no tiene un impacto de significancia para la satisfacción laboral. Sobre esta misma línea, Garcia (2003) realizó una investigación que tenía por objetivo pricipal constatar la existencia de beneficios no monetarios de la educación sobre el nivel de satisfacción laboral, basándose en el argumento de que el bienestar es una proxy a la utilidad derivada del trabajo medida a través del nivel de satisfacción en el empleo. La autora desarrolla este objetivo a través del empleo de modelo multinomiales ordenados, con datos obtenidos de la Plan de Hogares de la Unión Europea, para España, en el Año 1998.

En igual sentido, Clark (1996) realiza un estudio con el objeto de identificar los determinantes de la satisfacción laboral, distintos al salario, en Gran Bretaña. Incorpora a su análisis características demográficas como la edad, nivel educativo y estado civil y aspectos laborales como horas de trabajo, estatus sindical e inseguridad laboral. El autor encuentra una relación débil entre los ingresos y la satisfacción laboral, los hombres casados son los más satisfechos con su trabajo, y el 66\% de las mujeres británicas están satisfechas con su empleo, muestra que a mayor asociación sindical hay mayor insatisfacción laboral.

También, existen estudios que relacionan la calidad del empleo y la satisfacción laboral, como es el caso de Iglesias, Llorente y Dueñas (2010) para España. Los resultados evidencian que los madrileños tienen una alta calidad de empleo, pero son quienes menos satisfechos se sienten en sus trabajos, en relación con otras regiones españolas. Otras variables determinantes son la formación y la promoción, la salud y seguridad en el trabajo, la flexibilidad laboral y el diálogo social.

Para el caso de Colombia, Baquero, et al. (2018) si bien no hallaron los determinantes de la satisfacción en el empleo, lograron caracterizar la satisfacción laboral en las diferentes áreas metropolitanas de Colombia, en el marco del observatorio laboral LaboUR. Encuentran que Cartagena presenta los mayores índices de satisfacción, a diferencia de Pasto que tiene el menor índice. Otras ciudades con altas tasas de satisfacción en el empleo son Bucaramanga y Barranquilla. La diferencia en la satisfacción entre ciudades tiene que ver principalmente con los beneficios $y$ prestaciones que ofrecen las empresas a sus empleados y no tanto, al número de horas que trabajan. También observan que al 11.7\% de los encuestados en todas las áreas metropolitanas se sienten inconforme con su trabajo y que al $22 \%$ de los asalariados le gustaria cambiar de empleo. Angulo, Quejada y Yanez (2014), Barrera y Botia (2014) y Botello y Rios (2014), estiman diferentes tipos de modelos econométricos de elección discreta, con el fin de identificar los principales determinantes de la satisfacción laboral. Utilizando datos de corte transversal, encontraron 
que características propias del empleo, el sector donde laboran, las condiciones contractuales e inclusive la compatibilidad del trabajo con otras labores son variables con impacto positivo sobre la satisfacción laboral.

Por su parte, Amezquita (2013) busca probar que en conjunto, la calidad de empleo y la satisfacción laboral, reflejan las condiciones laborales, pero lo hacen desde apreciaciones distintas. Finalmente, corrobora su hipótesis y encuentra que la correlación entre calidad de empleo y satisfacción es significativa. También, plantea que la probabilidad que los colombianos tengan un empleo de calidad es cercana al 50\% y que la probabilidad de que estén satisfechos con éste es aproximadamente del 96\%. Ruíz (2013), identifica qué factores demográficos y cuáles características extrínsecas al trabajo se relacionan con la satisfacción laboral. Para alcanzar este objetivo utiliza modelos de tipo no lineal (probit Binario y Ordenado) y con datos de la Gran Encuesta Integrada de Hogares del año 2010, y obtiene como resultados que el salario y trabajar en el sector público, inciden positivamente en la satisfacción laboral, a diferencia de la variable horas laboradas y que el lugar de trabajo esté en lugares al aire libre, que afectan negativamente esta satisfacción.

Olarte (2011) en su trabajo tiene como objetivo integrar las diferentes disciplinas y proponer un conjunto de factores que agrupen los principales determinantes de la satisfacción laboral, a partir de una encuesta aplicada por el Ministerio de Educación en el 2010, como parte del programa del Observatorio Laboral que hace seguimiento a los graduados. En el modelo tomó factores demográficos, el nivel educativo y factores extrínsecos (salario y tipo de contrato) e intrínsecos (dificultad del trabajo y relaciones interpersonales). Los resultados obtenidos muestran que la percepción del trabajo en el crecimiento profesional y la relación directa del trabajo con la profesión, fueron las variables que tenían mayor relación con la satisfacción en el empleo, variables como el tipo de vinculación y el ingreso también son importantes pero en menor proporción. Frente a la educación, se encontró que tiene una relación inversa con la satisfacción laboral.

De otro lado, Farné y Vergara (2007) abordan la problemática de la calidad de empleo en Colombia y verifican si los indicadores objetivos de la calidad de empleo se ven evidenciados en la evaluación que los individuos hacen de su empleo. Confirman lo que se espera a partir de la teoría económica, a mayor ingreso, mayor satisfacción laboral y a más horas de trabajo, mayor insatisfacción. Muestran también que, en Colombia, están menos satisfechos los trabajadores con estado civil separados y con niveles altos de educación. Las mujeres, los trabajadores cuenta propia formales y los asalariados del sector público están satisfechos con su empleo. La deslaboralización, por su parte, (temporalidad, subcontratación, ausencia de contrato de trabajo o cuenta propia informal), genera un impacto negativo en la satisfacción de los individuos con su trabajo.

Finalmente, en el caso de la ciudad de Santiago de Cali, son pocos los estudios que analizan la satisfacción laboral. Solo se cuenta con el desarrollado por Rivas (2014) que busca exponer los determinantes de la satisfacción laboral para los trabajadores del sector informal, tomando los datos de la Encuesta de Empleo y Calidad de Vida -EECV- de año 2012. Para alcanzar este objetivo, aplicó un modelo de elección discreta (Probit), encontrando principalmente, que en el sector formal la satisfacción esta ligada principalmente a los horarios y no a la remuneración. Además de ello, una característica que reduce la probabilidad de encontrarse satisfecho en el sector informal es ser jefe de hogar y tener niveles educativos universitarios o de postgrado. También se tuvo en cuenta la variable pertenencia étnica, la cual no resulta ser significativa para los empleados informales. Por último, se tienen estudios de Arroyo, et al. (2016), González-Rivas (2012) y Bustamante \& Arroyo (2008), que no analizan propiamente la satisfacción laboral, sino la calidad y la discriminación en el mercado laboral de la ciudad de Cali. Desde este punto de vista, el presente trabajo contribuye a llenar el vacio empírico que hay en este este tipo de estudios, en particular, para estudios en el contexo local. 


\section{METODOLOGÍA}

Esta sección se presentan los fundamentos teóricos para la construcción del modelo econométrico objeto de análisis, luego se describe la naturaleza de los datos base del desarrollo de dicho modelo y, por último, se expondrán las variables relevantes para este estudio.

\subsection{El modelo}

Una de las formas de medir la satisfacción, es usando una escala de Likert, en este caso se toma un número de valores finitos y discretos que contienen información ordinal, siguiendo una escala establecida, donde el valor inferior puede ser "muy insatisfecho" y el superior "muy satisfecho", existiendo opciones intermedias entre estas dos categorías, por ende, es pertinente desarrollar un modelo para el análisis que tenga en cuenta esta característica de tipo ordinal. De acuerdo con Rodríguez Donate y Cáceres Hernández (2007), este tipo de modelos supone la existencia de una variable latente no observada ${ }^{1} y^{*}$, la cual está en función de un conjunto de variables independientes, de la siguiente manera:

$$
y_{i}^{*}=X_{i} \beta+\varepsilon_{i}
$$

Donde, $X_{i}$ es una matriz que contiene las variables independientes, $\beta$ un vector de coeficientes del modelo, $\varepsilon_{\mathrm{i}}$ son los residuos. Según Ruíz Tavera (2013) la variable indicadora $y_{i}$ es función de la variable latente $y_{i}^{*}$ además de una serie de puntos de corte que definen el orden del modelo. Esta relación se da de la siguiente manera:

$$
y_{i}=j \quad \text { si } \quad \tau_{(j-1)} \leq y_{i}^{*}<\tau_{j}, \quad j=1, \ldots, J
$$

De manera extendida se puede escribir:

$$
y_{i}=\left\{\begin{array}{lll}
1 & \text { si } & \tau_{0} \leq y^{*}{ }_{i}<\tau_{1} \\
2 & \text { si } & \tau_{1} \leq y^{*}{ }_{i}<\tau_{2} \\
& & \ldots \\
j & \text { si } & \tau_{j-1} \leq y^{*}{ }_{i}<\tau_{j} \\
j & \text { si } & \tau_{j-1} \leq y^{*}{ }_{i}<\tau_{j}
\end{array}\right.
$$

Donde $\tau_{0}=-\infty$ y $\tau_{j}=\infty$. La variable latente $y_{i}^{*}$ puede estar en un rango que va desde $-\infty$ a $\infty$. El $\tau$, son los umbrales o puntos de corte de la variable $y_{i}^{*}$, que dividen su recorrido en intervalos asociados con las distintas alternativas de $y_{i}$. Estos umbrales son parámetros poblacionales no observables, pero sí estimables y considerando que la variable respuesta es de tipo ordinal, se debe cumplir que,

1 Las variables latentes son aquellas que no se observan directamente, sino que son inferidas mediante otras variables observables y medibles directamente, estas últimas se denominan variables indicadoras. 


$$
\tau_{1}<\tau_{2}<\cdots<\tau_{(J-1)}
$$

Con $j$, el número de niveles considerados, siendo $1=$ completa insatisfacción y $j=$ máxima satisfacción. Cuando $j$ es 2, la ecuación [1.4] es igual a un modelo de elección binaria (Long,1997 citado en Ruíz Tavera, 2013, pág. 107)]. Si $y_{i}{ }^{*}<\tau_{1}$ el individuo i selecciona la alternativa 1 , si $\tau_{1} \leq y_{i}{ }^{*}$ $<\tau_{2}$ el individuo selecciona la alternativa 2, y así hasta llegar a la última alternativa J; entonces, se necesitarán tantos puntos de corte como alternativas haya, menos uno (Bustamante Romani, 2014).

En la propuesta planteada en este trabajo, la variable latente es el bienestar subjetivo del trabajo $\left(B S T_{i}\right)$, la cual está en función de un conjunto variables independientes que determinan el nivel de satisfacción de individuo con su empleo actual, siendo la variable indicadora la satisfacción laboral. Teniendo en cuenta lo anterior, la ecuación [1.1] se puede escribir así:

$$
B S T_{i}=X_{i}^{\prime} \beta+\varepsilon_{i}
$$

Donde, $B S T_{i}$ el vector de respuestas que contiene los niveles de satisfacción laboral respecto al empleo actual, $X_{i}^{\prime}$ corresponde al vector de características asociadas con el individuo en el actual empleo, $\beta$ es un vector columna que contiene los coeficientes del modelo, los cuales miden la influencia o relación de las variables independientes sobre la dependiente, $y \varepsilon_{-} i$ es un vector columna con n residuos, es también conocido como error aleatorio. La relación existente entre la variable ordinal satisfacción laboral del individuo i (sat.), que consta de cuatro niveles, y la variable latente continua bienestar subjetivo del trabajo $\left(B S T_{i}\right)$, que indica el grado de satisfacción del individuo frente al trabajo, es como sigue:

$$
\begin{aligned}
& \text { sat }_{i}=1 \rightarrow \text { Muy Insatisfecho si }-\infty \leq B S T_{i}<\tau_{1} \\
& \text { sat }_{i}=2 \rightarrow \text { Insatisfecho } \quad \text { si } \quad \tau_{1} \leq \text { BSTi }<\tau_{2} \\
& \text { sat }_{i}=3 \rightarrow \text { Satisfecho si } \tau_{2} \leq \text { BSTi }<\tau_{3} \\
& \text { sat }_{i}=4 \rightarrow \text { Muy Satisfecho si } \tau_{3} \leq \text { BSTi }<\infty
\end{aligned}
$$

Así, la Satisfacción Laboral expresada por el individuo i, se interpreta como un indicador que se genera a partir de la información contenida en el Bienestar Subjetivo del Trabajo. La escala considerada para que el individuo valore su nivel de satisfacción consta, en este trabajo, de 4 niveles, a saber:

$$
\text { sat }_{i}=j \quad \text { si } \quad \tau_{(j-1)} \leq B S T_{i}<\tau_{j}, \quad j=1, \ldots, 4
$$

Donde $\tau$, son los umbrales de la variable $B S T_{i}$ que dividen su recorrido en intervalos asociados con las puntuaciones de $s a t_{i}, j$, los niveles de satisfacción considerados, $s_{a} t_{i}=j$ si el individuo elige la alternativa $\mathrm{j}$. Las alternativas deben ser mutuamente excluyentes y exhaustivas, esto es solo puede escoger una y deben ser consideradas todas las opciones posibles. Considerando la variable indicadora $y_{i}$, se puede expresar de la siguiente forma, reemplazando [1.1] en [1.3]:

Dejando los residuales en un solo lado de la ecuación:

$$
y_{i}=\left\{\begin{array}{cccc}
1 & \text { si } & -\infty \leq X_{i}^{\prime} \beta+\varepsilon_{i}<\tau_{1} \\
2 & \text { si } & \tau_{1} \leq X_{i}^{\prime} \beta+\varepsilon_{i}<\tau_{2} \\
& & \ldots & \\
j & \text { si } & \tau_{(j-1)} \leq X_{i}^{\prime} \beta+\varepsilon_{i}<\tau_{j} \\
j & \text { si } & \tau_{(J-1)} \leq X_{i}^{\prime} \beta+\varepsilon_{i}<\infty
\end{array}\right.
$$




$$
\mathrm{y}_{\mathrm{i}}=\left\{\begin{array}{ccc}
1 & \text { si } & -\infty-X_{i}^{\prime} \beta \leq \varepsilon_{i}<\tau_{1}-X_{i}^{\prime} \beta \\
2 & \text { si } & \tau_{1}-X_{i}^{\prime} \beta \leq \varepsilon_{i}<\tau_{2}-X_{i}^{\prime} \beta \\
& \ldots \\
j & \text { si } & \tau_{(j-1)}-X_{i}^{\prime} \beta \leq \varepsilon_{i}<\tau_{j}-X_{i}^{\prime} \beta \\
j & \text { si } & \tau_{(J-1)}-X_{i}^{\prime} \beta \leq \varepsilon_{i}<\infty-X_{i}^{\prime} \beta
\end{array}\right.
$$

En este tipo de modelos, el objetivo es encontrar la probabilidad de que un acontecimiento suceda. El modelo probabilístico que determina la elección de cada alternativa:

$$
\begin{aligned}
P\left(y_{i}=j \mid X\right) & =P\left(\tau_{(j-1)}<y_{i}^{*} \leq \tau_{j}\right)=P\left(\tau_{(j-1)}<X_{i}{ }^{\prime} \beta+\varepsilon_{i} \leq \tau_{j}\right) \\
& =P\left(\tau_{(j-1)}-X_{i}^{\prime} \beta<\varepsilon_{i} \leq \tau_{j}-X_{i}^{\prime} \beta\right) F\left(\tau_{j}-X_{i}{ }^{\prime} \beta\right)-F\left(\tau_{(j-1)}-X_{i}^{\prime} \beta\right)
\end{aligned}
$$

Entonces, la probabilidad de cada alternativa es:

$$
\begin{aligned}
P\left(y_{i}=1 \mid X\right) & =P\left(-\infty-X_{i}{ }^{\prime} \beta \leq \varepsilon_{i}<\tau_{1}-X_{i}^{\prime} \beta\right)=F\left(\tau_{1}-X_{i}^{\prime} \beta\right)-F\left(-\infty-X_{i}^{\prime} \beta\right)=F\left(\tau_{1}-X_{i}^{\prime} \beta\right) \\
P\left(y_{i}=2 \mid X\right) & =P\left(\tau_{1}-X_{i}^{\prime} \beta \leq \varepsilon_{i}<\tau_{2}-X_{i}^{\prime} \beta\right)=F\left(\tau_{2}-X_{i}^{\prime} \beta\right)-F\left(\tau_{1}-X_{i}^{\prime} \beta\right) \\
& \cdots \\
P\left(y_{i}=j \mid X\right) & =P\left(\tau_{(j-1)}-X_{i}^{\prime} \beta \leq \varepsilon_{i}<\tau_{j}-X_{i}^{\prime} \beta\right)=F\left(\tau_{j}-X_{i}^{\prime} \beta\right)-F\left(\tau_{(j-1)}-X_{i}^{\prime} \beta\right) \\
P\left(y_{i}=J \mid X\right) & =P\left(\tau_{(J-1)}-X_{i}^{\prime} \beta \leq \varepsilon_{i}<\infty-X_{i}^{\prime} \beta\right)=F\left(\infty-X_{i}^{\prime} \beta\right)-F\left(\tau_{(J-1)}-X_{i}^{\prime} \beta\right) \\
& =1-F\left(\tau_{(J-1)}-X_{i}^{\prime} \beta\right)
\end{aligned}
$$

Donde $\mathrm{F}$ es la función de distribución acumulada ${ }^{2} F(\bullet)$ de la variable aleatoria $\varepsilon_{i}$. La distribución del error aleatorio determina el tipo de modelo a utilizar. Dentro de las distribuciones más utilizadas están la logística y la normal, que dan lugar a un modelo ordenado logit y probit ${ }^{3}$ respectivamente. Bajo ambas distribuciones, la metodología de estimación es por máxima verosimilitud. Si $\varepsilon_{i}$ sigue una distribución normal estándar, se tiene el modelo probit ordenado $F(\bullet)=\Phi(\bullet)$, donde $\Phi(\bullet)$ simboliza la función de distribución normal estándar. Si $\varepsilon_{i}$ sigue una distribución logística, se tiene el modelo logit ordenado $F(\bullet)=\Lambda(\bullet)$, donde $\Lambda(\bullet)$ simboliza la función de distribución logística. Nótese que cuando $y_{i}=1$, el término $F\left(-\infty-X_{i}^{\prime} \beta\right)=0, y$ cuando $y_{i}=J$, el término $F\left(\infty-X_{i}^{\prime} \beta\right)=1^{4}$. Si se utiliza el modelo logit ordenado, la función quedaría expresada así:

$$
F\left(\tau_{j}-X_{i}^{\prime} \beta\right)=\Lambda\left(\tau_{j}-X_{i}^{\prime} \beta\right)=e^{\left(\tau_{j}-X_{i}^{\prime} \beta\right)} /\left(1+e^{\left(\tau_{j}-X_{i}^{\prime} \beta\right)}\right), \forall j=1,2, \ldots, J-1
$$

Si se utiliza el modelo probit ordenado, la función quedaría expresada así:

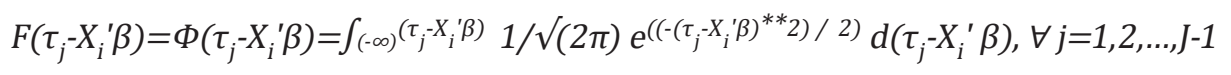

\footnotetext{
2 Sea $X$ es una variable aleatoria discreta con rango de valores $R_{X}$, entonces su función de probabilidad se define por: $p(x)=P[X=x]$, $\forall x \in R_{x} ; p(x)>0 ; \sum p(x)=1$. Sea $X$ una variable aleatoria discreta con función de probabilidad $p(x)$ y rango de valores $R_{X}$, entonces su función de distribución acumulativa se define por: $F(t)=P(X \leq t)=\sum_{(x \leq t)} p(x)$ donde t escualquier número real entero no negativo, entonces: $F(X)=p(0)+p(1)+p(2)+\ldots+p(t)$.
}

3 Las funciones logit y probit son simétricas; sin embargo, las colas de la función logit tiene colas más anchas por lo que tiende a dar mayor probabilidad a los extremos de $Y$ cuando $Y^{*}$ es más grande, o más pequeño, comparada con la probit (González Arbeláez, 2010).

4 En general una distribución acumulativa $F(x)$ de una variable aleatoria discreta es una función no decreciente de los valores de $X$, de tal manera que: $0 \leq F(x) \leq 1$ (Para valores extremos de $x$, como en nuestro caso $-\infty$ o $\infty$, la probabilidad es 00 1 , según sea al caso); $F\left(x_{i}\right) \geq F\left(x_{j}\right)$ si $x_{i} \geq x_{j}$ y $P(X>X)=1-F(x)$. 
En la metodología de los modelos ordenados, los coeficientes estimados $\beta$ no son los efectos marginales, estos se pueden estimar para cada uno de los niveles calculando el cambio parcial de las probabilidades y estimando los cambios marginales de cada variable. El efecto marginal de una variable continua es:

$$
\left(\delta P\left(y_{i}=j \mid X\right) /\left(\delta X_{i k}\right)\right)=\left[F^{\prime}\left(\tau_{(j-1)}-X_{i}^{\prime} \beta_{i}\right)-F^{\prime}\left(\tau_{j}-X_{i}^{\prime} \beta_{i}\right)\right] \beta_{k}
$$

De esta manera, el efecto marginal es la pendiente de la curva que relaciona a $X_{i k}$ con $P\left(y_{i}=j\right)$, manteniendo lo demás constante. Uno de los supuestos de este modelo es el de líneas paralelas o proportional odds, el cual indica que los coeficientes estimados $\beta$ son iguales para todas las categorías de la variable dependiente. Si la hipótesis no se cumple, se puede solucionar con la aplicación del Test de Brand para conocer cuáles son las variables que no cumplen con el supuesto, para después utilizar otros modelos como los logit ordenados generalizados (González Arbeláez, 2010).

\section{2. $\quad$ Naturaleza de los datos}

La modelación econométrica que se desarrollará en el presente trabajo se hará a partir de la información recolectada en la Encuesta de Empleo y Calidad de Vida para el Municipio de Santiago de Cali (EECV), realizada por el Ministerio de Trabajo y la Alcaldía de Santiago de Cali (Colombia), entre noviembre 2012 a enero 2013. Esta se desarrolló con el propósito de entender las problemáticas del mercado laboral de esta ciudad, se aplicó a 8.600 hogares y 30.458 personas, en 22 comunas en el sector urbano y una en el área rural, con un error de muestreo esperado no superior a $1.2 \%$ para el total Cali y no superior a $7 \%$ para los resultados en cada una de las comunas. De la base de datos resultante, el enfoque principal se hace sobre las variables contenidas en el módulo IX: Mercado de Trabajo.

\subsection{Variable relevantes}

La satisfacción laboral se medirá a partir de la pregunta: “Está muy satisfecho, satisfecho, insatisfecho o muy insatisfecho con su trabajo actual?" (pregunta $\mathrm{N}^{\circ} 81$, Módulo IX-b, EECV), que dentro del modelo econométrico corresponde a la variable dependiente; mientras las variables independientes son aquellas pertenecientes a los grupos de variables demográficas y extrínsecas al trabajo. Dentro del primer grupo, teniendo en cuenta que uno de los enfoques de este trabajo es identificar diferencias en la satisfacción laboral, según la variable de autorreconocimiento étnico, para controlar por este factor se incluirá de dos formas, la primera variable incluirá las categorías: indígena, negra, mulata, blanca, mestiza, otra, no sabe; la segunda, una variable dummy en la regresión, la cual toma el valor de 1 si el trabajador se autorreconoce de etnia negra y 0 en otro caso.

Para la inclusión de las variables extrínsecas del trabajo, se construirá un índice de la calidad del empleo (ICE), basado en el índice sintético propuesto por Farné (2003), fundamentado en cuatro variables básicas: el ingreso, la modalidad de contratación, la afiliación a la seguridad social y el horario de trabajo. El rango de valores propuestos por la OIT está entre 0 y 100, considerando empleos de buena calidad aquellos que el índice toma valores superiores a 60 puntos, lo que indica que entre más alto sea el puntaje obtenido por el trabajador, se considera que este tiene un empleo de mayor calidad. La construcción de este índice se constituye como un aporte adicional en este trabajo, ya que es otro factor diferenciador de las demás investigaciones realizados en este ámbito. A continuación, se describen las variables utilizadas en el modelo: 


\subsubsection{Variable dependiente.}

Satisfacción laboral: mide el nivel de satisfacción del individuo con su trabajo actual, es una variable cualitativa ordenada con 4 categorías: "1. Muy satisfecho", "2. Satisfecho", "3. Insatisfecho"

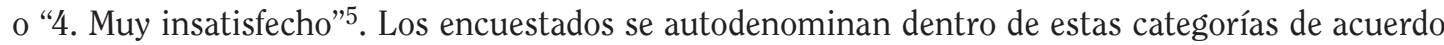
con su percepción. Corresponde a la pregunta $\mathrm{N}^{\circ}$ 81, Módulo IX-b "Mercado de trabajo, ocupados" de la EECV.

\subsubsection{Variables independientes demográficas.}

Raza: indica el autorreconocimiento étnico de la persona. Es una variable categórica con siete alternativas a saber, indígena, negra, mulata, blanca, mestiza, otra, no sabe. La categoría base es blanca, ya que ha sido demostrado en estudios anteriores que ser de raza blanca aumenta las probabilidades de conseguir empleo y el salario promedio de las personas, al ser comparadas con las de otras razas. Corresponde a la pregunta $\mathrm{N}^{\circ}$ 13 del Módulo III.

Estrato: corresponde al nivel socioeconómico de la vivienda, contenido en el Módulo "Identificación", los niveles van desde el 1 hasta el 6. Estrato base para el modelo es el 4. Pregunta $\mathrm{N}^{\circ} 11$ Módulo Identificación.

Edad: se establecen 6 rangos, los grupos se conforman así: menores de 18 años, 18 a 24 años, 25 a 35 años, 36 - 45 años, 46 - 55 años, mayores de 55 años. Edad base para el modelo es la de 18 a 24 años. Se encuentra en el Módulo II.

Género: variable dicotómica, toma dos valores, 1 si es Hombre y 0 si es Mujer. Pregunta que hace parte de la información demográfica contenida en el Módulo II.

Estado civil: clasifica en seis categorías, siendo estas unión libre, viudo/separadodivorciado, soltero, casado. Ser soltero se toma como base para el modelo. Pregunta $\mathrm{N}^{\circ}$ 9 Módulo II.

Jefe del hogar: información referida al parentesco con el jefe del hogar, dado esto, se incluye una variable dicotómica agrupando dos categorías así: 1 si el individuo es del hogar y 0 en otro caso. Pregunta $\mathrm{N}^{\circ} 6$ Módulo II.

Nivel educativo: variable ordinal categórica, corresponde al nivel educativo más alto alcanzado. Se agrupa de la siguiente manera, ninguno, bachiller, tecnólogo, universitario, especialización, master o doctorado. La categoría base para el modelo es ninguna educación. Pregunta $\mathrm{N}^{\circ} 9$ Módulo VII.

\subsubsection{Variables independientes extrínsecas del trabajo.}

Medio de transporte: variable ordinal categórica que se refiere al medio de transporte utilizado para ir al sitio de trabajo. Las categorías son: MIO, bus buseta o colectivo, automóvil de uso particular, taxi, pirata (carro particular que presta servicio de taxi), mototaxi, motocicleta, bicicleta, transporte de la empresa, a pie, bus intermunicipal, otro. Categoría base para el modelo: automóvil de uso particular. Pregunta $\mathrm{N}^{\circ} 64$ Módulo IX-b.

Tiempo de transporte: variable ordinal categórica, corresponde al tiempo en minutos que tarda el individuo en llegar a su lugar de trabajo desde su hogar. Pregunta $\mathrm{N}^{\circ} 65$ Módulo IX-b.

5 La escala original de esta pregunta se recodifica, esto se hace para que el valor inferior se asocie con el nivel mínimo nivel de satisfacción (1. Muy insatisfecho) y el más alto con el máximo nivel de satisfacción (4. Muy satisfecho). 
ICE: (Índice de Calidad de Empleo): construido a partir de las variables: ingreso, modalidad de contratación, afiliación a la seguridad social y horario de trabajo. Esta es una variable cuantitativa continua que toma valores entre 0 y 100 . Las variables se asocian con las siguientes preguntas de la EECV, Módulo IX-b: ingreso asalariados, pregunta $\mathrm{N}^{\circ} 18$; ingreso independientes, pregunta $\mathrm{N}^{\circ} 40$; modalidad de contratación, pregunta $\mathrm{N}^{\circ} 4$ y 5 ; afiliación a seguridad social, pregunta $\mathrm{N}^{\circ} 52$; horario de trabajo, pregunta $\mathrm{N}^{\circ} 46$. En el cuadro 1 , se describen las variables involucradas en la construcción del índice, los puntajes y la ponderación según el tipo de ocupado (asalariado o independiente).

\section{CUADRO 1 \\ ÍNDICE DE CALIDAD DE EMPLEO PROPUESTO POR FARNÉ (2003)}

\begin{tabular}{|c|c|c|c|}
\hline & Puntos & Asalariado & Independiente \\
\hline Ingreso & & $40 \%$ & $50 \%$ \\
\hline Menos de 1.5 SMMLV & 0 & & \\
\hline Entre 1.5 y 3 SMMLV & 50 & & \\
\hline Más de 3 SMMLV & 100 & & \\
\hline Modalidad de contratación & & $25 \%$ & \\
\hline Ausencia de contrato laboral & 0 & & \\
\hline Contrato laboral a término fijo & 50 & & \\
\hline Contrato laboral a término indefinido & 100 & & \\
\hline Afiliación a seguridad social & & $25 \%$ & $35 \%$ \\
\hline Sin afiliación a salud o pensión & 0 & & \\
\hline Afiliado a salud o pensión & 50 & & \\
\hline Afiliado a salud y pensión & 100 & & \\
\hline Horario de trabajo & & $10 \%$ & $15 \%$ \\
\hline Horario de más de 48 horas semanales & 0 & & \\
\hline Horario de hasta de 48 horas semanales & 100 & & \\
\hline
\end{tabular}

Fuente: Tomado de Bustamante y Arroyo Mina (2008). 


\section{RESULTADOS}

Se plantea un modelo econométrico que se ajuste a la información objeto de estudio, basándose principalmente en los modelos logit ordinal y probit ordinal, los cuales consideran una variable categórica ordenada como dependiente. Estos modelos permiten identificar las variables independientes que explican los niveles de satisfacción de los individuos con su empleo, más precisamente con los empleados asalariados de la Ciudad de Cali que participaron en la EECVSC. El modelo se estructura con 7.496 observaciones, las cuales cuentan con información en todas las variables que se analizan.

La variable dependiente puede tomar cuatro valores que se relacionan con la satisfacción que siente el individuo con su empleo actual, entre mayor sea el valor de la variable, la persona está más satisfecha con este. Según los datos, el grupo con mayor participación es el de aquellas que se sienten satisfechas con su trabajo (68,94\%). Es importante encontrar un modelo que diferencie las alternativas y que permita identificar los aspectos que son relevantes para que los individuos se sientan satisfechos o no con su empleo. En el cuadro 2, se muestra la frecuencia de cada uno de los niveles de esta variable.

\section{CUADRO 2}

FRECUENCIA DE LA VARIABLE DEPENDIENTE SATISFACCIÓN LABORAL

\begin{tabular}{lrrr}
\hline & Frecuencia & Porcentaje & Acumulado \\
\hline Muy insatisfecho & 95 & $1,27 \%$ & $1,27 \%$ \\
Insatisfecho & 1.230 & $16,41 \%$ & $17,68 \%$ \\
Satisfecho & 5.168 & $68,94 \%$ & $86,62 \%$ \\
Muy satisfecho & 1.003 & $13,38 \%$ & $100,00 \%$ \\
Total & 7.496 & $100 \%$ &
\end{tabular}

Fuente: Cálculos propios con base en Encuesta de Empleo y Calidad de Vida -EECV- 2012-2013.

Como se mencionó, se considerarán los modelos logit ordenado y probit ordenado, donde Sat es la variable dependiente, $y$ dentro del conjunto de variables explicativas se encuentra el grupo de variables demográficas que son raza (autorreconocimiento étnico), estrato, edad, género, estado civil, jefe de hogar (parentesco con el jefe del hogar), nivel educativo y luego tenemos el grupo de variables extrínsecas del trabajo, estas son ICE, medio (medios de transporte), tiempo (de viaje al trabajo). La estructura de ambos modelos es la siguiente:

$$
\begin{array}{r}
\text { Sat }_{i}=\beta_{1} \text { raza }_{i}+\beta_{2} \text { estrato }_{i}+\beta_{3} \text { edad }_{i}+\beta_{4} \text { género }_{i}+\beta_{5} \text { estado }_{\text {civili }}+\beta_{6} \text { jefe }_{\text {hogari }}+ \\
\beta_{7} \text { nivel }_{\text {educativoi }}+\beta_{8} \text { ice }_{i}+\beta_{9} \text { medio }_{i}+\beta_{10} \text { tiempo }_{i}+\varepsilon_{i}
\end{array}
$$

En el cuadro 3, se muestran las estimaciones realizadas con los modelos logit ordinal y probit ordinal. Los coeficientes, aunque diferentes, mantienen su signo y significancia, lo que posiblemente permitiría llegar a la misma conclusión en ambos casos. Con base en los resultados, se encontró que las variables que no aportan significativamente a la construcción del modelo son: género, jefe de hogar, nivel educativo y tiempo de viaje, por tanto, se propone estimar un nuevo modelo sin incluir estas variables (ver cuadro 4). El objetivo de estimar un segundo modelo es encontrar un modelo parsimonioso, dado que si un modelo incluye solo los componentes que sean relevantes, tendrá la capacidad de predecir mejor. En el tabla 4, se encuentran los resultados del modelo reducido, con las variables que son estadísticamente significativas. 
Es necesario encontrar el modelo que mejor se ajuste a los datos, esto es, que haga mejores predicciones. Para definir cuál es el más adecuado, dentro de un número finito de modelos, se consideran los criterios de información, estos son una importante herramienta para la selección de modelos econométricos. El objetivo de estos criterios es calcular una medida que refleje qué tan cercanos están los modelos propuestos al verdadero modelo que genera los datos, lo que permite seleccionar como "óptimo" aquel modelo cuyo criterio de información sea más pequeño. Los criterios más usados son: el de Akaike (AIC, Akaike Information Criterion) y el de Schwarz o bayesiano (BIC, Bayesian Information Criterion). Se calcularon estos criterios de información para cada modelo propuesto, resultando que el modelo logit de la segunda estimación presenta menores valores de AIC y BIC, así, se considera este modelo para el análisis de este trabajo.

CUADRO 3

\section{ESTIMACIÓN DE LA SATISFACCIÓN LABORAL (LOGIT ORDENADO VS PROBIT ORDENADO)}

\begin{tabular}{|c|c|c|c|c|c|c|}
\hline \multirow{2}{*}{$\begin{array}{l}\text { Variable dependiente } \\
\text { Satisfacción Laboral }\end{array}$} & \multicolumn{3}{|c|}{ Modelo logit ordenado } & \multicolumn{3}{|c|}{ Modelo probit ordenado } \\
\hline & Coeficiente & & Error estándar & Coeficiente & & Error estándar \\
\hline \multicolumn{7}{|l|}{ Raza considerada } \\
\hline Indígena & $-0,2913$ & $* * *$ & 0,1064 & $-0,1696$ & $* * *$ & 0,0586 \\
\hline Negra & $-0,2279$ & $* * *$ & 0,0872 & $-0,1120$ & $* * *$ & 0,0478 \\
\hline Mulata & $-0,2499$ & $* * *$ & 0,0866 & $-0,1350$ & $* * *$ & 0,0473 \\
\hline Mestiza & $-0,1274$ & $* *$ & 0,0616 & $-0,0709$ & $* *$ & 0,0337 \\
\hline Otra & 0,7551 & & 1,4669 & 0,4107 & & 0,8698 \\
\hline No sabe & 0,0605 & & 0,1333 & 0,0113 & & 0,0721 \\
\hline \multicolumn{7}{|l|}{ Estrato } \\
\hline 1 & $-0,2878$ & $* * *$ & 0,0770 & $-0,1552$ & $* * *$ & 0,0421 \\
\hline 2 & $-0,1455$ & $* *$ & 0,0633 & $-0,0759$ & $* *$ & 0,0344 \\
\hline 4 & 0,4161 & $* * *$ & 0,1306 & 0,2556 & $* * *$ & 0,0733 \\
\hline 5 & 0,2172 & $* *$ & 0,1006 & 0,1286 & $* *$ & 0,0554 \\
\hline 6 & 0,5930 & $* * *$ & 0,1515 & 0,3489 & $* * *$ & 0,0857 \\
\hline \multicolumn{7}{|l|}{ Edad } \\
\hline Menor de 18 & 0,2001 & & 0,3110 & 0,1370 & & 0,1740 \\
\hline $26-35$ & 0,0954 & & 0,0843 & 0,0546 & & 0,0462 \\
\hline $36-45$ & 0,1707 & $*$ & 0,0897 & 0,0887 & $*$ & 0,0490 \\
\hline $46-55$ & 0,1598 & $*$ & 0,0943 & 0,0863 & $*$ & 0,0517 \\
\hline Más de 55 & 0,2415 & $* *$ & 0,1090 & 0,1243 & $* *$ & 0,0596 \\
\hline \multicolumn{7}{|l|}{ Género } \\
\hline Mujer & 0,0781 & & 0,0581 & 0,0374 & & 0,0319 \\
\hline \multicolumn{7}{|l|}{ Estado civil } \\
\hline Unión libre & 0,1302 & $*$ & 0,0702 & 0,0691 & $*$ & 0,0384 \\
\hline Viudo/Separado-Divorciado & $-0,0465$ & & 0,0889 & $-0,0175$ & & 0,0485 \\
\hline Casado & 0,1602 & $* *$ & 0,0756 & 0,0877 & $* *$ & 0,0415 \\
\hline \multicolumn{7}{|l|}{ Jefe del hogar } \\
\hline Jefe(a) del hogar & 0,0312 & & 0,0593 & 0,0143 & & 0,0325 \\
\hline
\end{tabular}




\begin{tabular}{|c|c|c|c|c|c|c|}
\hline \multirow{2}{*}{$\begin{array}{l}\text { Variable dependiente } \\
\text { Satisfacción Laboral }\end{array}$} & \multicolumn{3}{|c|}{ Modelo logit ordenado } & \multicolumn{3}{|c|}{ Modelo probit ordenado } \\
\hline & Coeficiente & & Error estándar & Coeficiente & & Error estándar \\
\hline \multicolumn{7}{|l|}{ Nivel educativo } \\
\hline Bachiller & 0,0369 & & 0,0635 & 0,0150 & & 0,0347 \\
\hline Tecnólogo & 0,0201 & & 0,1020 & 0,0077 & & 0,0555 \\
\hline Universitario & $-0,0775$ & & 0,1019 & $-0,0452$ & & 0,0561 \\
\hline Espec., Master o Doctorado & $-0,1132$ & & 0,1639 & $-0,0828$ & & 0,0924 \\
\hline ICE & 0,0181 & $* * *$ & 0,0012 & 0,0098 & $* * *$ & 0,0006 \\
\hline \multicolumn{7}{|l|}{ Medio de transporte } \\
\hline MIO & $-0,2027$ & * & 0,1050 & $-0,1132$ & * & 0,0582 \\
\hline Bus, buseta o colectivo & $-0,4678$ & **** & 0,1155 & $-0,2616$ & **** & 0,0636 \\
\hline Taxi & $-0,2995$ & & 0,2190 & $-0,2007$ & & 0,1210 \\
\hline Pirata & $-0,2075$ & & 0,2537 & $-0,1490$ & & 0,1393 \\
\hline Mototaxi & $-0,3845$ & & 0,3521 & $-0,2296$ & & 0,1936 \\
\hline Motocicleta & $-0,2577$ & ** & 0,1116 & $-0,1533$ & ** & 0,0617 \\
\hline Bicicleta & $-0,3773$ & $* * *$ & 0,1368 & $-0,2048$ & $* * *$ & 0,0747 \\
\hline Transporte de la empresa & $-0,0983$ & & 0,1840 & $-0,0670$ & & 0,1005 \\
\hline A pie & $-0,2826$ & $* * *$ & 0,1100 & $-0,1577$ & $* * *$ & 0,0608 \\
\hline Bus intermunicipal & $-0,3555$ & & 0,4431 & $-0,1940$ & & 0,2355 \\
\hline Otro & $-0,1264$ & & 0,2783 & $-0,0447$ & & 0,1507 \\
\hline Tiempo de viaje & $-0,0018$ & & 0,0012 & $-0,0008$ & & 0,0007 \\
\hline Cut1 & $-4,0531$ & & 0,1785 & $-2,1015$ & & 0,0893 \\
\hline Cut2 & $-1,1725$ & & 0,1493 & $-0,7226$ & & 0,0818 \\
\hline Cut3 & 2,5574 & & 0,1532 & 1,4599 & & 0,0831 \\
\hline Observaciones & 7496 & & & 7496 & & \\
\hline LR Chi2(38) & 745,8800 & & & 743,9200 & & \\
\hline Prob > Chi2 & 0,0000 & & & 0,0000 & & \\
\hline Pseudo R2 & 0,0567 & & & 0,0566 & & \\
\hline Log likelihood & $-6204,3937$ & & & $-6205,3740$ & & \\
\hline
\end{tabular}

***Coeficiente estadísticamente significativo al 1\%, **5\%, *10\%.

Fuente: Cálculos propios con base en EECV, 2012-2013. 


\section{CUADRO 4: \\ ESTIMACIÓN DE LA SATISFACCIÓN LABORAL (MODELO REDUCIDO)}

\begin{tabular}{|c|c|c|c|c|c|c|}
\hline \multirow{2}{*}{$\begin{array}{l}\text { Variable dependiente } \\
\text { Satisfacción Laboral }\end{array}$} & \multicolumn{3}{|c|}{ Modelo logit ordenado } & \multicolumn{3}{|c|}{ Modelo probit ordenado } \\
\hline & Coeficiente & & Error estándar & Coeficiente & & Error estándar \\
\hline \multicolumn{7}{|l|}{ Raza considerada } \\
\hline Indígena & $-0,2970$ & $* * *$ & 0,1063 & $-0,1714$ & $* * *$ & 0,0585 \\
\hline Negra & $-0,2298$ & $* * *$ & 0,0870 & $-0,1130$ & $* *$ & 0,0477 \\
\hline Mulata & $-0,2499$ & $* * *$ & 0,0865 & $-0,1347$ & $* * *$ & 0,0472 \\
\hline Mestiza & $-0,1314$ & $* *$ & 0,0615 & $-0,0728$ & $* *$ & 0,0337 \\
\hline Otra & 0,7667 & & 1,4654 & 0,4161 & & 0,8694 \\
\hline No sabe & 0,0569 & & 0,1332 & 0,0092 & & 0,0720 \\
\hline \multicolumn{7}{|l|}{ Estrato } \\
\hline 1 & $-0,3035$ & $* * *$ & 0,0754 & $-0,1610$ & $* * *$ & 0,0412 \\
\hline 2 & $-0,1502$ & $* *$ & 0,0628 & $-0,0775$ & $* *$ & 0,0341 \\
\hline 4 & 0,4052 & $* * *$ & 0,1298 & 0,2491 & $* * *$ & 0,0729 \\
\hline 5 & 0,2036 & $* *$ & 0,0987 & 0,1205 & $* *$ & 0,0545 \\
\hline 6 & 0,5674 & $* * *$ & 0,1492 & 0,3328 & $* * *$ & 0,0844 \\
\hline \multicolumn{7}{|l|}{ Edad } \\
\hline Menor de 18 & 0,1768 & & 0,3098 & 0,1262 & & 0,1733 \\
\hline $26-35$ & 0,0914 & & 0,0836 & 0,0520 & & 0,0458 \\
\hline $36-45$ & 0,1681 & $*$ & 0,0875 & 0,0868 & $*$ & 0,0478 \\
\hline $46-55$ & 0,1534 & * & 0,0906 & 0,0822 & * & 0,0497 \\
\hline Más de 55 & 0,2244 & $* *$ & 0,1030 & 0,1161 & $* *$ & 0,0563 \\
\hline \multicolumn{7}{|l|}{ Estado civil } \\
\hline Unión libre & 0,1360 & $* *$ & 0,0681 & 0,0717 & $*$ & 0,0372 \\
\hline Viudo/Separado-Divorciado & $-0,0156$ & & 0,0854 & $-0,0025$ & & 0,0466 \\
\hline Casado & 0,1612 & ** & 0,0744 & 0,0877 & $* *$ & 0,0408 \\
\hline ICE & 0,0177 & $* * *$ & 0,0011 & 0,0096 & $* * *$ & 0,0006 \\
\hline \multicolumn{7}{|l|}{ Medio de transporte } \\
\hline MIO & $-0,1985$ & $*$ & 0,1020 & $-0,1087$ & $*$ & 0,0566 \\
\hline Bus, buseta o colectivo & $-0,4545$ & $* * *$ & 0,1131 & $-0,2529$ & $* * *$ & 0,0623 \\
\hline Taxi & $-0,2711$ & & 0,2182 & $-0,1859$ & & 0,1207 \\
\hline Pirata & $-0,1812$ & & 0,2529 & $-0,1330$ & & 0,1388 \\
\hline Mototaxi & $-0,3717$ & & 0,3508 & $-0,2216$ & & 0,1932 \\
\hline Motocicleta & $-0,2281$ & $* *$ & 0,1088 & $-0,1372$ & $* *$ & 0,0603 \\
\hline Bicicleta & $-0,3779$ & $* * *$ & 0,1342 & $-0,2018$ & $* * *$ & 0,0733 \\
\hline Transporte de la empresa & $-0,0952$ & & 0,1821 & $-0,0633$ & & 0,0996 \\
\hline A pie & $-0,2099$ & $* *$ & 0,1047 & $-0,1214$ & $* *$ & 0,0580 \\
\hline Bus intermunicipal & $-0,4415$ & & 0,4376 & $-0,2367$ & & 0,2332 \\
\hline Otro & $-0,0398$ & & 0,2753 & $-0,0042$ & & 0,1493 \\
\hline
\end{tabular}




\begin{tabular}{lrrrr} 
Variable dependiente & \multicolumn{2}{c}{ Modelo logit ordenado } & \multicolumn{2}{c}{ Modelo probit ordenado } \\
\cline { 2 - 5 } \multicolumn{1}{c}{ Satisfacción Laboral } & Coeficiente & Error estándar & Coeficiente & Error estándar \\
\hline Cut1 & $-4,0471$ & 0,1661 & $-2,0967$ & 0,0818 \\
Cut2 & $-1,1683$ & 0,1343 & $-0,7192$ & 0,0738 \\
Cut3 & 2,5589 & 0,1386 & 1,4624 & 0,0752 \\
& & & & \\
Observaciones & 7496 & & 7496 & \\
LR Chi2(38) & 739.89 & & 0,0000 & \\
Prob > Chi2 & 0,0000 & & 0,0562 & \\
Pseudo R2 & 0,0562 & & $-6207,8849$ & \\
Log likelihood & $-6207,3903$ & & & \\
\hline
\end{tabular}

*** Coeficiente estadísticamente significativo al 1\%, ** $5 \%$, *10\%.

Fuente: Cálculos propios con base en EECV, 2012-2013.

Considerando que el modelo previamente seleccionado es el logit ordenado del cuadro 4, se debe validar el supuesto de líneas paralelas. Para ello se utiliza el Test de Brant, cuya hipótesis nula es que los coeficientes estimados $\beta$ son iguales para todas las categorías de la variable dependiente. Los resultados muestran que no hay suficiente evidencia para rechazar la hipótesis nula a nivel de todas las variables, ni a nivel específico, de esta manera, se puede decir que el modelo propuesto cumple con el supuesto de líneas paralelas y por tanto es un modelo adecuado (ver cuadro 5).

\section{CUADRO 5: \\ TEST DE BRANT PARA PROBAR LA HIPÓTESIS DE LÍNEAS PARALELAS.}

\begin{tabular}{lrrr}
\hline Variable & Chi2 & P-Value Chi2 & Df \\
\hline Todas & 6,7 & 0,876 & 12 \\
Raza considerada & 1,3 & 0,519 & 2 \\
Estrato & 0,6 & 0,738 & 2 \\
Edad & 0,8 & 0,686 & 2 \\
Estado civil & 0,2 & 0,921 & 2 \\
ICE & 2,1 & 0,353 & 2 \\
\hline
\end{tabular}

Fuente: Cálculos propios con base en EECV- 2012-2013.

En este tipo de modelos los coeficientes no permiten conocer una medida de influencia sobre la variable dependiente, sin embargo, el signo indica la relación con la probabilidad de estar en la categoría más alta y su signo contrario con la probabilidad de estar en la categoría más baja. Esto es, un coeficiente con signo positivo, indica que la variable independiente asociada tiene una relación positiva con la categoría más alta y negativa con la categoría más baja de la variable dependiente. Las estimaciones se muestran en el cuadro 4 y los efectos marginales en el cuadro 6. El primero muestra la dirección del efecto de las variables sobre la satisfacción laboral y el segundo permite cuantificar este efecto sobre cada una de las categorías.

Como el objetivo específico del presente trabajo es identificar cuál el efecto que tiene el autorreconocimiento étnico, sobre la satisfacción laboral en la ciudad de Santiago de Cali, la variable raza toma gran importancia dentro del análisis. La categoría base de dicha variable es "blanca", el 
signo del coeficiente estimado indica que, con relación a las personas que se consideran blancas, los individuos que se autorreconocen de otra raza tienen una relación negativa respecto a estar muy satisfecho con el trabajo actual, y están más propensos a estar insatisfechos con este. Los efectos marginales reflejan que el impacto sobre la probabilidad de encontrarse insatisfecho con el empleo actual es positivo, si se compara a las personas que se autorreconocen indígena, negra, mulata $o$ mestiza, con aquellas que se autorreconocen blancas. Es decir, ser de una raza diferente a la blanca, influye de manera positiva en la probabilidad de encontrarse insatisfecho con su trabajo actual. Por ejemplo, autoreconocerse como negro, aumenta la probabilidad de estar muy insatisfecho con el empleo en $0,28 \%$, con respecto a los blancos, y así mismo, reduce la probabilidad de estar muy satisfecho en $0,247 \%$, en relación con los que se autoreconocen como blancos. Estas relaciones de autoreconocimiento, tanto en la situación "muy insatisfecho" como "muy satisfecho", se mantienen en las demás razas: el que el individuo se autoreconozca como indigena, mulato o meztizo, aumenta la probabilidad de estar muy insatisfecho, $y$ reduce la probabilidad de estar muy satisfecho con el empleo actual, todo, en comparación con los blancos (ver cuadro 6).

\section{CUADRO 6 \\ EFECTOS MARGINALES SOBRE LA PROBABILIDAD DE ENCONTRARSE SATISFECHO CON EL EMPLEO}

\begin{tabular}{|c|c|c|c|c|c|c|c|c|}
\hline \multirow{2}{*}{$\begin{array}{c}\text { Variable dependiente } \\
\text { Satisfacción Laboral }\end{array}$} & \multicolumn{8}{|c|}{ Modelo logit ordenado } \\
\hline & \multicolumn{2}{|c|}{ Muy insatisfecho } & \multicolumn{2}{|c|}{ Insatisfecho } & \multicolumn{2}{|c|}{ Satisfecho } & \multicolumn{2}{|c|}{ Muy satisfecho } \\
\hline \multicolumn{9}{|l|}{ Raza considerada } \\
\hline Indígena & 0,0038 & $* *$ & 0,0379 & $* * *$ & $-0,0104$ & $*$ & $-0,0313$ & **** \\
\hline Negra & 0,0028 & ** & 0,0288 & $* * *$ & $-0,0069$ & * & $-0,0247$ & $* * *$ \\
\hline Mulata & 0,0031 & *** & 0,0315 & **** & $-0,0079$ & $* *$ & $-0,0267$ & $* * *$ \\
\hline Mestiza & 0,0015 & ** & 0,0160 & $* *$ & $-0,0030$ & $* *$ & $-0,0146$ & ** \\
\hline Otra & $-0,0059$ & & $-0,0709$ & & $-0,0341$ & & 0,1109 & \\
\hline No sabe & $-0,0006$ & & $-0,0066$ & & 0,0005 & & 0,0067 & \\
\hline \multicolumn{9}{|l|}{ Estrato } \\
\hline 1 & 0,0040 & *** & 0,0401 & $* * *$ & $-0,0139$ & **** & $-0,0302$ & $* * *$ \\
\hline 2 & 0,0018 & ** & 0,0190 & $* *$ & $-0,0051$ & $* *$ & $-0,0157$ & ** \\
\hline 4 & $-0,0038$ & $* * *$ & $-0,0434$ & $* * *$ & $-0,0037$ & & 0,0510 & $* * *$ \\
\hline 5 & $-0,0021$ & $* *$ & $-0,0232$ & $* *$ & 0,0013 & & 0,0240 & ** \\
\hline 6 & $-0,0049$ & $* * *$ & $-0,0578$ & $* * *$ & $-0,0123$ & & 0,0750 & $* * *$ \\
\hline \multicolumn{9}{|l|}{ Edad } \\
\hline Menor de 18 & $-0,0023$ & & $-0,0227$ & & 0,0064 & & 0,0185 & \\
\hline $26-35$ & $-0,0012$ & & $-0,0120$ & & 0,0039 & & 0,0093 & \\
\hline $36-45$ & $-0,0022$ & * & $-0,0216$ & * & 0,0062 & * & 0,0176 & * \\
\hline $46-55$ & $-0,0020$ & * & $-0,0198$ & * & 0,0059 & & 0,0160 & * \\
\hline Más de 55 & $-0,0028$ & ** & $-0,0284$ & $* *$ & 0,0074 & $* *$ & 0,0239 & ** \\
\hline \multicolumn{9}{|l|}{ Estado civil } \\
\hline Unión libre & $-0,0017$ & * & $-0,0172$ & $* *$ & 0,0044 & * & 0,0145 & ** \\
\hline Viudo/Separado-Divorciado & 0,0002 & & 0,0020 & & $-0,0007$ & & $-0,0016$ & \\
\hline Casado & $-0,0020$ & ** & $-0,0202$ & $* *$ & 0,0049 & ** & 0,0174 & $* *$ \\
\hline
\end{tabular}




\begin{tabular}{|c|c|c|c|c|c|c|c|c|}
\hline \multirow{3}{*}{$\begin{array}{l}\begin{array}{c}\text { Variable dependiente } \\
\text { Satisfacción Laboral }\end{array} \\
\text { ICE }\end{array}$} & \multicolumn{8}{|c|}{ Modelo logit ordenado } \\
\hline & \multicolumn{2}{|c|}{ Muy insatisfecho } & \multicolumn{2}{|c|}{ Insatisfecho } & \multicolumn{2}{|c|}{ Satisfecho } & \multicolumn{2}{|c|}{ Muy satisfecho } \\
\hline & $-0,0002$ & $* * *$ & $-0,0022$ & $* * *$ & 0,0005 & $* * *$ & 0,0019 & $* * *$ \\
\hline \multicolumn{9}{|l|}{ Medio de transporte } \\
\hline MIO & 0,0021 & ** & 0,0231 & ** & $-0,0020$ & & $-0,0232$ & * \\
\hline Bus, buseta o colectivo & 0,0055 & $* * *$ & 0,0568 & **** & $-0,0134$ & $* * *$ & $-0,0490$ & $* * *$ \\
\hline Taxi & 0,0030 & & 0,0322 & & $-0,0042$ & & $-0,0310$ & \\
\hline Pirata & 0,0019 & & 0,0209 & & $-0,0016$ & & $-0,0213$ & \\
\hline Mototaxi & 0,0043 & & 0,0454 & & $-0,0086$ & & $-0,0411$ & \\
\hline Motocicleta & 0,0025 & ** & 0,0267 & $* *$ & $-0,0028$ & & $-0,0264$ & $* *$ \\
\hline Bicicleta & 0,0044 & $* * *$ & 0,0462 & $* * *$ & $-0,0090$ & $*$ & $-0,0417$ & $* * *$ \\
\hline Transporte de la empresa & 0,0010 & & 0,0107 & & $-0,0002$ & & $-0,0115$ & \\
\hline A pie & 0,0023 & ** & 0,0245 & *** & $-0,0023$ & & $-0,0244$ & * \\
\hline Bus intermunicipal & 0,0054 & & 0,0550 & & $-0,0126$ & & $-0,0477$ & \\
\hline Otro & 0,0004 & & 0,0044 & & 0,0001 & & $-0,0049$ & \\
\hline
\end{tabular}

$* * *$ Coeficiente estadísticamente significativo al 1\%, **5\%, *10\%.

Fuente: Cálculos propios con base en EECV, 2012-2013.

Respecto a los otros posibles determinantes estudiados, los estratos socioeconómicos en los que se pueden clasificar las viviendas $y / o \operatorname{los}_{\text {predios }}{ }^{6}$ donde residen los individuos entrevistados, muestran que, en relación con el estrato medio-bajo (estrato 3) los estratos inferiores son los más propensos a encontrarse insatisfechos con su trabajo, además disminuyen en 3,02\% para el estrato 1 y 1,57\% para el 2 la probabilidad de sentirse muy satisfechos, mientras que los estratos superiores como medio, medio alto $y$ alto aumenta las probabilidades de estar satisfecho $y$ muy satisfecho con su actividad laboral. Esta relación negativa para los estratos inferiores se puede dar porque tienen acceso limitado a los empleos de buena calidad o porque para cubrir sus necesidades básicas deben hacer lo que esté a su alcance, $y$ posiblemente esto no coincida con sus preferencias.

Desde el punto de vista de la edad, el grupo más joven, después de cumplida la mayoría de edad (18 a 25 años), y pertenecer a un grupo de mayor edad (superior a 36 años) aumenta las probabilidades de estar en un empleo donde se sienta satisfecho desarrollando sus labores. Es posible que, en la primera etapa laboral, las personas jóvenes estén explorando diferentes campos en la búsqueda de la labor que les agrada, mientras los adultos en muchos casos ya tienen definida esta situación, además de seguramente poder acceder a mejores salarios dada su mayor experiencia laboral. Ahora bien, las personas que conviven de forma estable mediante matrimonio o unión parecen tener una mayor probabilidad de encontrarse más a gusto con su oficio que las personas que son solteras. Estar en unión libre aumenta la probabilidad de sentirse satisfecho con su trabajo en un $1,45 \%$ y estar casado la aumenta en un $1,74 \%$.

El Índice de Calidad de Empleo -ICE-, que se fundamenta en cuatro dimensiones: ingreso, modalidad de contratación, afiliación a la seguridad social y Horario de trabajo, es una medición cuantitativa que toma valores entre 0 y 100. Se esperaría que la relación entre el ICE y el nivel de satisfacción fuera positiva. Esta hipótesis se valida con los resultados encontrados, en los efectos marginales se encuentra que, si el ICE de un individuo aumenta en 1 unidad, la probabilidad de estar en el nivel superior de satisfacción con su labor aumenta $0,19 \%$.

6 De acuerdo al DANE, los estratos socioeconómicos en los que se pueden clasificar las viviendas y/o los predios son 6, denominados así: 1. Bajo-bajo; 2. Bajo; 3. Medio-bajo; 4. Medio; 5. Medio-alto; 6. Alto. 
El medio en que se transporta la población de Calí a su sitio de trabajo incide en la satisfacción que este tiene con su empleo. Las personas que se desplazan en transporte masivo MIO, bus-busetacolectivo, motocicleta, bicicleta y a pie tienen una mayor probabilidad de sentirse insatisfechos con su empleo, respecto a aquellos que viajan en automóvil de uso particular, en otras palabras, los individuos que se desplazan en transporte público, moto, bicicleta o que van a caminando a su empleo, tienen una menor probabilidad de encontrarse entusiasmados con su ocupación.

Desplazarse en MIO (sistema de transporte masivo) reduce la probabilidad en 2,32\% de estar en el máximo nivel de satisfacción, igual ocurre con el bus-buseta-colectivo que la disminuye en $4,90 \%$, motocicleta en $2,64 \%$, bicicleta en $4,17 \%$ y a pie $2,44 \%$. Esto refleja que poder transportarse en automóvil particular tiene un impacto positivo sobre la satisfacción en el empleo. En todos los casos, los niveles intermedios de satisfacción se comportan similar a su nivel más próximo, es decir, el nivel insatisfecho se comporta de manera similar a muy insatisfecho y a su vez, el nivel satisfecho tiene un comportamiento parecido a muy satisfecho.

Ahora bien, deseando conocer qué pasa específicamente con las personas afrodescendientes, dada la alta proporción en la ciudad de Cali, se construyó una variable dicotómica o dummy, la cual toma el valor de 1 si el trabajador se autorreconoce de raza negra, y 0 en otro caso. Se incorporó y se estimó nuevamente el modelo, cuyos resultados se ilustran en el cuadro 7. Como se ve, esta variable no resultó estadísticamente significativa, por lo que se concluye que, la raza negra por si sola, no es un factor condicionante de la satisfacción laboral. Esto sugiere que la influencia de la raza negra sobre la satisfacción laboral, solo se presenta cuando se consideran los demás grupos raciales ${ }^{7}$.

\section{CUADRO 7 \\ ESTIMACIÓN DE LA SATISFACCIÓN LABORAL (CON RAZA DICOTÓMICA)}

\begin{tabular}{lcrr}
\hline \multirow{2}{*}{ Muestras } & \multicolumn{2}{c}{ Modelo Logit Ordenado (Raza categórica } \\
dicotómica)
\end{tabular}

7 Este mismo ejercicio se realizó para cada una de las categorias de la raza, es decir, se construyó una dummy para indigena, mulato y blanco, y se estimó el modelo para cada una de ellas. Los resultados dieron una relación significativa de cada una las variables, sobre la satisfacción laboral. Para indigena y mulato, hay una disminución en la probabiliad de sentirse muy satisfeccho con el actual empleo, respecto a las demás razas, y para blanco hay un aumento en dicha probabilidad. 


\begin{tabular}{|c|c|c|c|}
\hline \multirow[t]{2}{*}{ Muestras } & \multicolumn{3}{|c|}{$\begin{array}{l}\text { Modelo Logit Ordenado (Raza categórica } \\
\text { dicotómica) }\end{array}$} \\
\hline & Coeficiente & & $\overline{\text { tándar }}$ \\
\hline \multicolumn{4}{|l|}{ Estado civil } \\
\hline Unión libre & 0,1328 & * & 0,0680 \\
\hline Viudo/Separado-Divorciado & $-0,0131$ & & 0,0854 \\
\hline Casado & 0,1688 & $* *$ & 0,0743 \\
\hline ICE & 0,0177 & $* * *$ & 0,0011 \\
\hline \multicolumn{4}{|l|}{ Medio de transporte } \\
\hline MIO & $-0,2073$ & $* *$ & 0,1020 \\
\hline Bus, buseta o colectivo & $-0,4777$ & $* * *$ & 0,1129 \\
\hline Taxi & $-0,2683$ & & 0,2179 \\
\hline Pirata & $-0,2031$ & & 0,2525 \\
\hline Mototaxi & $-0,3845$ & & 0,3504 \\
\hline Motocicleta & $-0,2335$ & $* *$ & 0,1087 \\
\hline Bicicleta & $-0,3943$ & $* * *$ & 0,1340 \\
\hline Transporte de la empresa & $-0,1110$ & & 0,1821 \\
\hline A pie & $-0,2234$ & $* *$ & 0,1046 \\
\hline Bus intermunicipal & $-0,4570$ & & 0,4366 \\
\hline Otro & $-0,0440$ & & 0,2748 \\
\hline Cut1 & $-3,9522$ & & 0,1625 \\
\hline Cut2 & $-1,0753$ & & 0,1299 \\
\hline Cut3 & 2,6452 & & 0,1348 \\
\hline Observaciones & 7496 & & \\
\hline LR Chi2(38) & 724,2300 & & \\
\hline Prob > Chi2 & 0,0000 & & \\
\hline Pseudo R2 & 0,0551 & & \\
\hline Log likelihood & $-6215,2169$ & & \\
\hline
\end{tabular}

***Coeficiente estadísticamente significativo al 1\%, **5\%, *10\%.

Fuente: Cálculos propios con base en EECV, 2012-2013. 


\section{CONCLUSIONES}

El objetivo principal de este trabajo era identificar el efecto del autorreconocimiento étnico sobre la satisfacción laboral en la ciudad de Santiago de Cali, de igual manera, se pretendía conocer los principales determinantes de la satisfacción laboral, considerando variables de tipo demográficas y variables asociadas con la calidad del empleo. Para contestar estos objetivos, se empleó un modelo logit ordenado, usando los datos de la Encuesta de Empleo y Calidad de Vida -EECV- para el Municipio de Santiago de Cali, realizada por el Ministerio de Trabajo y la Alcaldía de Santiago de Cali, en el periodo noviembre de 2012 a enero de 2013.

La importancia de este análisis se fundamenta en que la satisfacción en el empleo es un tema de amplio interés en el mercado laboral, ya que está altamente relacionada con la productividad de los trabajadores en su labor, además de brindar información sobre la calidad de vida de estos, dado el gran número de horas que pasa un individuo en su trabajo. Igualmente, para el caso de Cali, el estudio toma mayor relevancia, al buscar identificar si autorreconocerse de raza negra implicaba una menor satisfacción laboral, teniendo en cuenta la gran población afrodescendiente de la Ciudad que, de acuerdo con el CENSO realizado por DANE en el año 2005, representaba, junto con la población mulata $y$ afrocolombiana, el $26,12 \%$ de los habitantes.

Con este fin se construye un nuevo modelo con una variable dummy que toma el valor de 1 para el grupo de afrodescendientes y 0 en otro caso, los resultados de este modelo aportan información específica para la raza negra, encontrándose que ser afrodescendiente no tiene incidencia en sentirse satisfecho o no con su empleo. Con lo anterior, no tenemos evidencia suficiente para aceptar la hipótesis de que hay una influencia del autorreconocimiento afrodescendiente sobre la satisfacción laboral de los individuos que habitan en la ciudad de Cali.

Pese a lo anterior, la variable raza si tiene un impacto sobre la satisfacción laboral, puesto que cuando se evalúan en conjunto los diferentes grupos étnicos, ser de una raza diferente a la blanca (indígena, negra, mulata, mestiza) sí tiene un efecto negativo sobre la satisfacción laboral si se toma como categoría base la raza blanca. En el caso particular de interés en este estudio, ser afrodescendiente aumenta la probabilidad en $0,28 \%$ de sentirse insatisfecho, mientras que reduce en $2,47 \%$ la probabilidad de sentirse muy satisfecho.

Finalmente, se observa que, además de la raza, los determinantes de la satisfacción laboral en Cali son: estrato, edad, estado civil, la calidad del empleo (medida a través del ICE) y el medio de transporte para desplazarse al trabajo. Se encontró que los trabajadores que pertenecen a estratos socioeconómicos inferiores y utilizan transporte público, motocicleta, bicicleta o van caminando al trabajo, tienen una mayor probabilidad de sentirse insatisfechos con su empleo. Finalmente, ser de estratos socioeconómicos altos, tener más de 36 años, ser casado o estar en unión libre y tener una mejor calidad de empleo permite tener mayor probabilidad de encontrarse en un trabajo con el que se siente muy satisfecho. 


\section{REFERENCIAS}

Amezquita Ospina, D. M. (2013). Satisfacción laboral y calidad del empleo en Colombia: un análisis conjunto para el segundo trimestre del año 2012 [Tesis de Grado, Universidad del Valle]. Biblioteca Digital Universidad del Valle. http://hdl.handle.net/10893/5675

Angulo Pico, G., Quejada Pérez, R., \& Yanez Contreras, M. (2014). Ingresos adecuados y satisfacción laboral: análisis probabilístico. Trabajo y Sociedad, 22, 141-160.

Arroyo Mina, J. S., Pinzón Gutiérrez, L. F., Mora, J. J., Gómez Jaramillo, D. A., \& Cendales, A. (2016). Afrocolombianos, discriminación y segregación espacial en la calidad del empleo para Cali. Cuadernos de Economía, 35 (69), 753-783. https://doi.org/10.15446/cuad.econ.v35n69.54347

Barrera Barrios, B. F., \& Botia Díaz, J. G. (2014). Determinantes de la satisfacción laboral en Colombia [Tesis de Grado, Universidad Industrial de Santander]. Biblioteca Universidad Industrial de Santander. https://uids-primo.hosted.exlibrisgroup.com/permalink/f/1gjmsqs/ uids_bucaramanga167152.

Borra Marcos, C., \& Gómez García, F. (2012). Satisfacción laboral y salario: ¿Compensa la renta laboral las condiciones no monetarias del trabajo? Revista de Economía Aplicada, XX(60), 25-51.

Botello Peñaloza, H. A., \& Ríos, G. (2014). Determinantes de la satisfacción en el empleo en el Área Metropolitana de Bucaramanga, 2008-2012. Libre Empresa, 11(1), 13-25. https://doi. org/10.18041/1657-2815/libreempresa.2014v11n1.3010

Bravo, M., Peiró, J., \& Rodríguez, I. (1996). La actividad laboral en su contexto. En J. Peiró, \& F. Prieto (Eds.), Satisfacción laboral. Tratado de psicología del trabajo (pp. 343-394). Síntesis.

Bustamante, C. D., \& Arroyo Mina, S. (2008). La raza como determinante del acceso a un empleo de calidad: un estudio para la ciudad de Cali. Revista Ensayos Sobre Politica Económica, 26(57), 130-175. https://doi.org/10.32468/espe.5703

Bustamante Romani, R. (2014, Noviembre). Modelos de Elección Nominal. Aplicaciones en STATA 14. (Serie Apuntes de Clase $\Omega B T N^{\circ} 9$ ). Universidad Nacional Mayor de San Marcos. https:// economia.unmsm.edu.pe/data/apu_cla/apu_clase.htm

Cassar, L. (2010). Quality of Employment and Job Satisfaction: Evidence From Chile. (Research in Progress Series, 17a). University of Zurich. Oxford Poverty \& Human Development Initiative. https://ophi.org.uk/research-in-progress-17a/

Chiang Vega, M. M., \& Ojeda Hidalgo, J. F. (2013). Estudio de la relación entre satisfacción laboral y desempeño de los trabajadores de las ferias libres. Contaduria y Administración, 58(2), 39-60. https://doi.org/10.1016/S0186-1042(13)71209-9

Clark, A. E. (1996). Job Satisfaction in Britain. British Journal of Industrial Relations, 34(3), 189217. https://doi.org/10.1111/j.1467-8543.1996.tb00648.x

Diano, A. (2016). Incidencia del racismo en la motivación y satisfacción laboral de trabajadores afrodescendientes [Tesis de grado, Universidad de la República de Uruguay]. Repositorio libre Colibri. https://hdl.handle.net/20.500.12008/7974

Farné, S. (2003). Estudio sobre la calidad del empleo en Colombia. (Serie Estudios de Economía Laboral en Países Andinos no. 5), Organización Internacional del Trabajo.

Farné, S., \& Vergara, C. A. (2007). Calidad del empleo: ¿Qué tan satisfechos están los colombianos con su trabajo? Coyuntura Social, 36, 51-70. https://hdl.handle.net/11445/1747

Gamero Burón, C. (2005). Análisis Microeconómico de la Satisfacción Laboral. Consejo Económico y Social del Reino de España, Colección de Estudios.

Gamero Burón, C. (2007). Satisfacción Laboral y Tipo de Contrato en España. Investigaciones Económicas, XXXI(3), 415-444.

García Mora, M. B. (2003). Efectos de la educación sobre los determinantes de la satisfacción laboral en España. Un análisis de los beneficios monetarios y no monetarios en el mercado de trabajo mediante modelos Logit Ordenados [Tesis doctoral, Universidad de Valencia]. Tesis Doctorals en Xarxa. https://hdl.handle.net/10803/9694 
González-Rivas, N. (2012). Discriminación salarial: un análisis entre mujeres afrocolombianas y no afrocolombianas en el área metropolitana de Cali. Revista Latinoamericana de Ciencias Sociales, Niñez y Juventud, 10(1), 563-578. SCIELO

González Arbeláez, Á. (2010). Determinantes del riesgo de crédito comercial en Colombia. (Reporte de Estabilidad Financiera, No. 45). Banco de la República. https://doi.org/10.32468/tef.45

Griffin, R., \& Bateman, T. (1986). Job satisfaction and organizational commitment. En C. L. Cooper, \& I. Robertson (Edits.), International Review Industrial and Organization Psychology (pp. 233-247). John Wiley \& Sons.

Iglesias Fernández, C., Llorente Heras, R., \& Dueñas Fernández, D. (2011). Calidad del empleo y satisfacción laboral en las regiones españolas. Un estudio con especial referencia a la Comunidad de Madrid. Investigaciones Regionales, 19, 25-49. https://hdl.handle. net/10486/669625

Katzel, R. (1964). Personal Values, Job Satisfaction, and Job Behaviour. In Borow H. (ed.) Man in a World of Work. Houghton Mifflin.

Lancaster, K. (1971). Consumer Demand: A New Approach. Columbia University Press.

Locke, E. (1976). The nature and causes of job satisfaction. Hanbook of industry and Organizational Psycology.

Long, J. (1997). Regression Models for Categorical and Limited Dependent Variables. Advanced Quantitative Techniques in the Social Sciences series. Thousand Oaks: Sage Publications.

Lydon, R., \& Chevalier, A. (2002). Estimates of the effect of wages on job satisfaction (Discussion Papers 0531). Centre for Economic Performance.

McConnell, C., \& Brue, S. (1997). Economía Laboral. McGrawHill.

Observatorio Laboral LaboUR (2018). Patrones de satisfacción con el empleo urbano en Colombia (Informe 5). Observatorio Laboral, Universidad del Rosario. https://www.labourosario. com/post/2018/04/27/informe-patrones-de-satisfacci\%C3\%B3n-con-el-empleo-urbano-encolombia

Olarte, M. (2011). Los determinantes de la satisfacción laboral: Una revisión empírica. Facultad de Ciencias Económicas - Universidad Jorge Tadeo Lozano. https://www.academia. edu/27324464/LOS_DETERMINANTES_DE_LA_SATISFACCIÓN_LABORAL_UNA_ REVISIÓN_TEÓRICA_Y_EMPÍRICA_MARİA_LILIANA_OLARTE_VARGGAS_DIRECTOR_ JEANNE_KELLY_RUIZ_TAVERA

Pastor Ramos, G. (2000). Conducta interpersonal. Ensayo de psicología social sistemática. Universidad Pontificia de Salamanca.

Price, J. L., \& Mueller, C. W. (1986). Absenteeism and turnover among hospital employees.JAI Press.

Pujol-Cols, L. J., \& Dabos, G. E. (2018). Satisfacción laboral: una revisión de la literatura acerca de sus principales determinantes. Estudios Gerenciales, 34(146), 3-18.

https://doi.org/10.18046/j.estger.2018.146.2809

Reinecke, G., \& Valenzuela, M. E. (2000). La calidad del empleo: un enfoque de género. En M. E. Valenzuela, \& G. Reinecke (Eds.), ¿Más y mejores empleos para las mujeres? La experiencia de los países del Mercosur y Chile (pp. 29-58). Organización Internacional del Trabajo -OIT-.

Rivas Oyuela, P. A. (2014). Determinantes de la Satisfacción laboral para el sector informal en Cali (2012) [Tesis de Grado, Universidad del Valle]. Repositorio Digital Univalle http://hdl.handle. net/10893/7614

Robbins, L. (1932). An essay on the nature and significance of economic science. Macmillan.

Robbins, S. P. (2004). Comportamiento Organizacional (10a ed.). Pearson Educación.

Rodríguez Donate, M. C., \& Cáceres Hernández, J. J. (2007). Modelos de elección discreta y especificaciones ordenadas: una reflexión metodológica. Estadística Española, 49(166), 451-471.

Rose, M. (2000). The job satisfaction data in the WERS98 employee survey. How far can I trust it? En M. Rose (Ed.), Work Centrality, Work Careers and Household: Let's Ask for Numbers. Economic and Social Research Council .

Ruíz Tavera, J. K. (2013). Algunos determinantes de la Satisfacción Laboral en Colombia. Economia y Región, 7(2), 91-118. https://revistas.utb.edu.co/index.php/economiayregion/article/view/53 
Scitovsky, T. (1976). The Joyless Economy. Oxford University Press.

Somarriba Arrechavala, N., Ramos Truchelo, G., Merino Llorente, M. C., \& Negro Macho, A. (2007). Un análisis de la calidad del empleo y de la satisfacción laboral un estudio para Castilla y León. Revista Universitaria de Ciencias del Trabajo, 8, 301-320. http://uvadoc.uva.es/ handle/10324/11357

Stiglitz, J. E. (2002). Globalism's Discontents. The American Prospect, 13(1), A16-A21.

Vuotto, M., \& Arzadun, P. (2007). El buen trabajo y la satisfacción laboral (Documento 59). Centro de estudios de Sociologia del Trabajo. 